\title{
P-0539- Effects of high-fat feeding during pre-pregnancy, pregnancy and lactation on pancreatic islets' insulin secretion in rat
}

\author{
R. Karbaschi ${ }^{1}, 2$, H. Zardooz ${ }^{2}$, F. Sadeghimahalli ${ }^{3}$, F. Khodagholi ${ }^{4}$, L. Dargahi ${ }^{5}$, R. Arian ${ }^{6}$
}

${ }^{1}$ Faculty of Nursing and Midwifery, Shahid Beheshti University of Medical Sciences, Tehran, Iran. ${ }^{2}$ Neurophysiology Research Center, Department of Physiology, School of Medicine, Shahid Beheshti University of Medical Sciences, Tehran, Iran.

${ }^{3}$ Eduction Development Center- Mazandaran University of Medical Sciences, Sari, Iran.

${ }^{4}$ Neuroscience Research Center, Shahid Beheshti University of Medical Sciences, Tehran, Iran.

${ }^{5}$ NeuroBiology Research Center, Shahid Beheshti University of Medical Sciences, Tehran, Iran.

${ }^{6}$ Department of Dentistry- Shahid Beheshti University of Medical Sciences, Tehran, Iran.
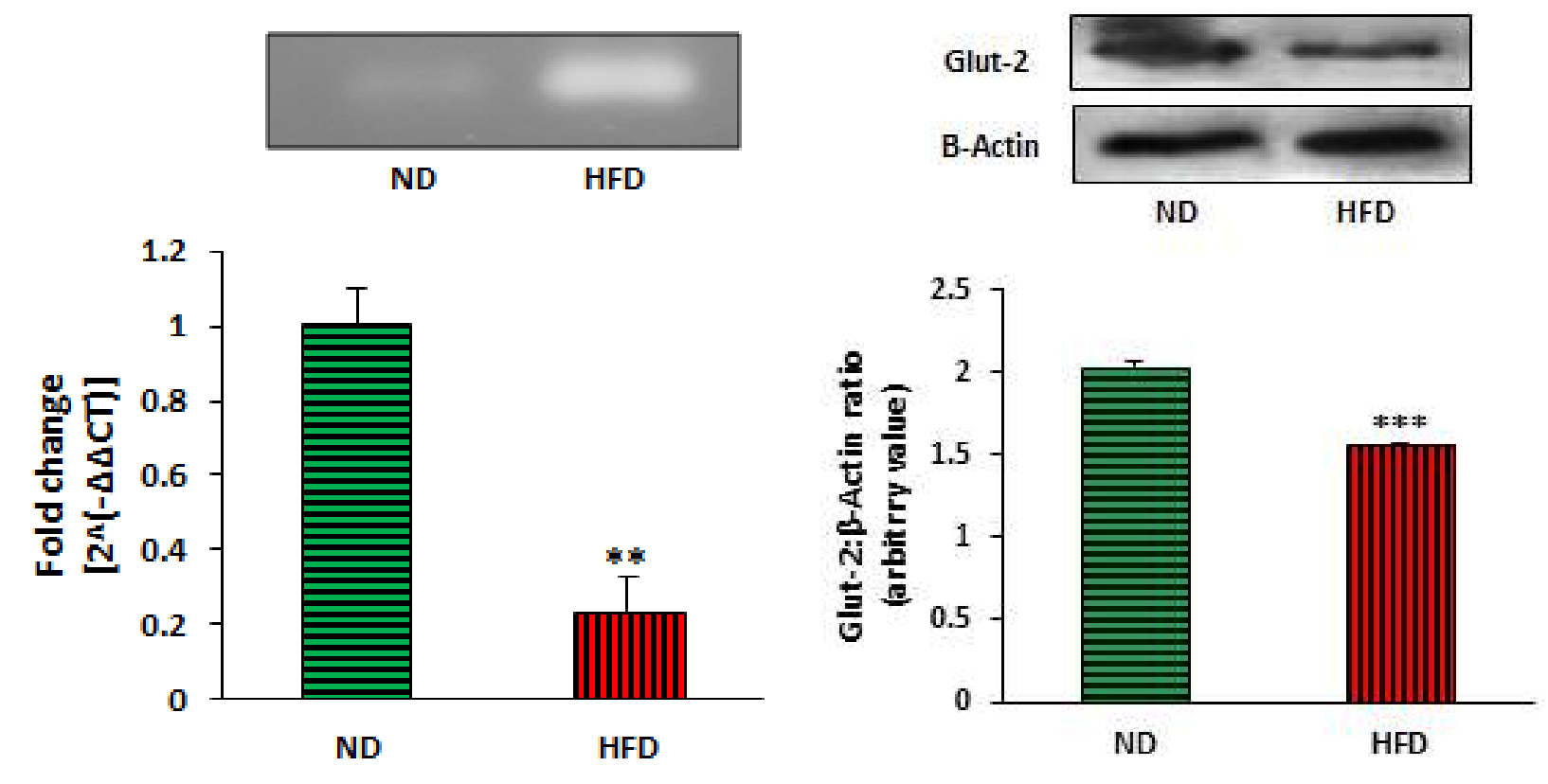

\section{Introduction}

The increasing prevalence of high-fat diets consumption is one of the main risk factors for metabolic diseases such as obesity and type 2 diabetes. It is noteworthy that most of the mothers desire to consume high-calorie and fat-rich diets in the gestation and lactation periods. The consumption of high-fat foods not only threatens the health of mothers and pregnancy process, but also by inducing metabolic impairment in dams, can increase the offspring's susceptibility to different disorders.

Considering the importance of this issue, this study was therefore conducted to examine the effect of a high-fat diet during prepregnancy, pregnancy and lactation on insulin secretion from isolated pancreatic islets in female Wistar rats.

\footnotetext{
Materials \& Methods

Animals were randomly divided into two groups of control (C) and high-fat (HF) diets and fed with either a normal or a high-fat diet during pre-pregnancy (4 weeks), pregnancy and lactation. The normal diet contained $2 \mathrm{~g} \%$ soybean oil, which provided $4.75 \% \mathrm{Kcal}$ as fat. The high fat diet, which was prepared by mixing $65 \mathrm{~g} \%$ ground standard pellets with $35 \mathrm{~g} \%$ cow butter, provided $58.2 \% \mathrm{Kcal}$ as fat (56.12 Kcal\% from cow butter and $2.08 \mathrm{Kcal} \%$ from soybean oil).

In both diet groups, at the end of lactation, blood sampling was performed by tail cutting under pentobarbital anesthesia, in fasting state. Then, the microtubes containing $5 \mu l$ heparin $(5000 \mathrm{IU} / \mathrm{ml})$ per 1 $\mathrm{ml}$ blood were centrifuged and plasma was separated and kept at $-70^{\circ} \mathrm{C}$ for measuring glucose and insulin concentrations.
}

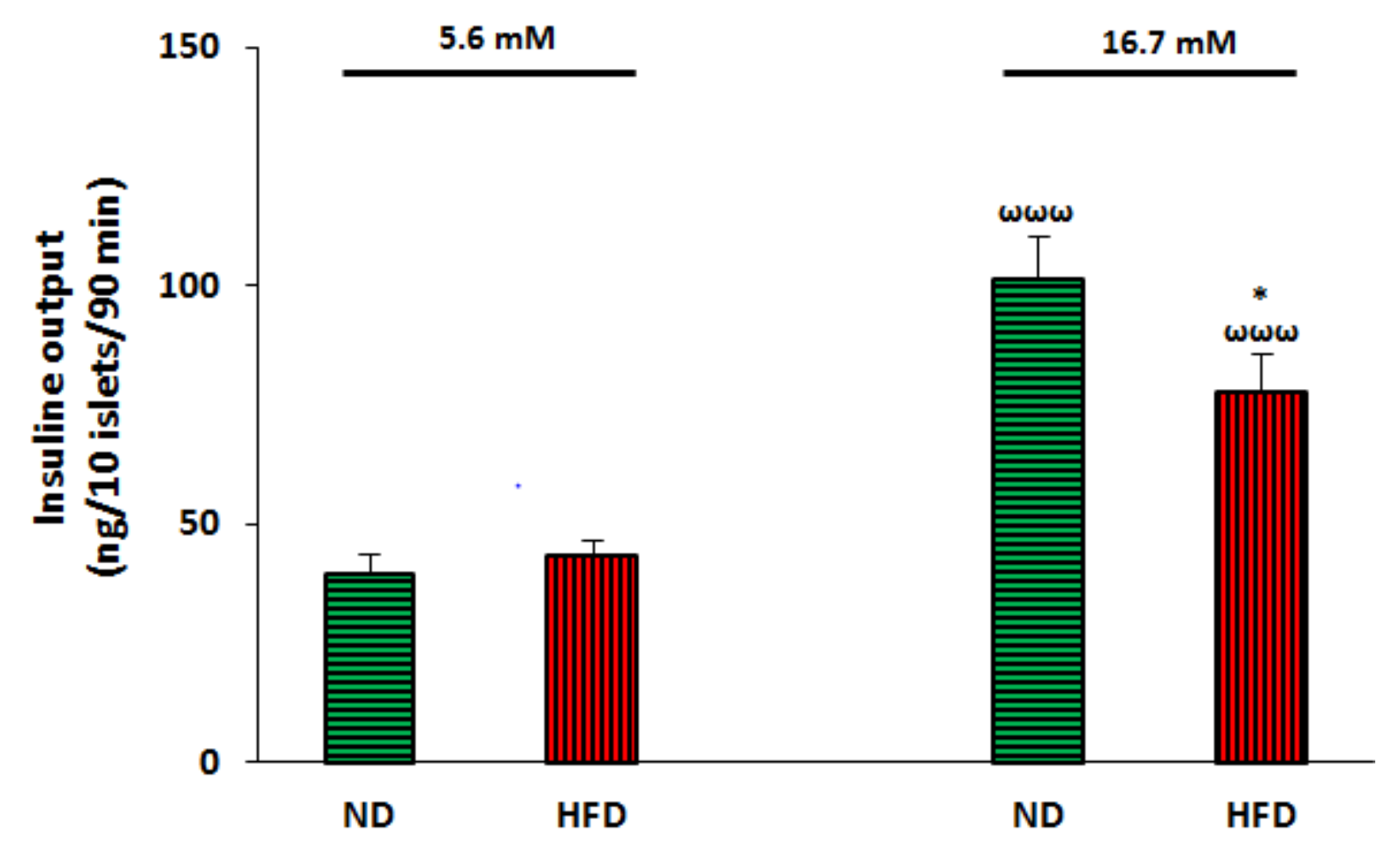

After blood sampling, the anesthetized animals were decapitated and the pancreasas was then removed for measuring insulin secretion from the isolated islets at 5.6 and $16.7 \mathrm{mM}$ glucose concentrations. Moreover, the pancrease tissue of the animals in both groups was used to assess glucose transporter $2 \mathrm{mRNA}$ expression and protein levels.

\section{Results}

In high-fat fed rats, insulin secretion from the isolated islets in the presence of $5.6 \mathrm{mM}$ glucose concentration did not significantly change, whereas the secretion decreased markedly at $16.7 \mathrm{mM}$ glucose concentration. Nevertheless, in HF group the plasma glucose and insulin concentrations remained unchanged in fasting state. On the other hand, the high-fat fed rats showed a significant decrease in glucose transporter 2 mRNA and protein expression.

\section{Discussion \& Conclusion}

According to the results of this study, the decrease in GLUT2 mRNA and protein expression which was observed in high-fat fed dams, did not affect insulin secretion from the isolated islets in response to basal $(5.6 \mathrm{mM})$ glucose concentration, which is in line with the results of in vivo experiment on fasting plasma insulin concentration. However, in these animals, the islet's insulin secretion ability was reduced in response to high glucose concentration $(16.7 \mathrm{mM})$. Thus it seems that high fat-diet consumption during pre-pregnancy, pregnancy and lactation could impair glucose stimulated insulin secretion, and therefore may affect glucose homeostasis.

\section{References}

1.Jacobs S, et al. Physiol behav. 2014;129:280-6.

2.Jacqueline L. Beaudry, Michael C. Riddell. Diabetes Metab Res Rev 2012; 28: 560-573.

3.Karbaschi et al. J Zhejiang Univ-Sci B (Biomed \& Biotechnol) 2016; 17(9):728-732.
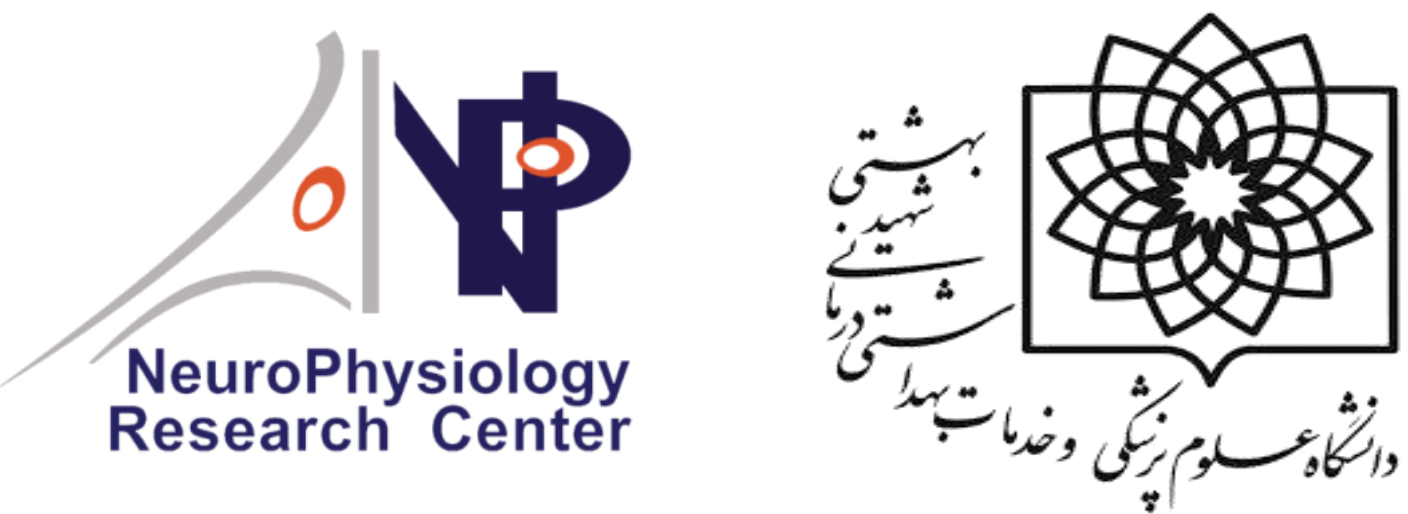